\title{
RETOS Y POTENCIALIDADES PARA LAS MUJERES EN LA PARTICIPACION DEL DESARROLLO DE LA SOCIEDAD DE LA INFORMACION
}

\author{
NÚRIA VERGÉS BOSCH \\ Programa Género y TIC. UOC. \\ Grupo de Investigación Cópolis. UB. \\ Colectivo de investigacción Donestech. Barcelona. \\ EVA CRUELLS LOPEZ \\ Colectivo de Investigacción Donestech. Barcelona \\ ALEX HACHE \\ Colectivo de Investigacción Donestech. Sevilla
}

Recibido: 01/07/2009

Aceptado: 01/09/2009

\section{Tecnologías y géneros: de las dicotomías a la fluidez}

A partir de finales de los años 70 creció, y se consolidó posteriormente, el interés por conocer las relaciones entre las mujeres y las tecnologías ${ }^{1}$. Este creciente interés cabe relacionarlo, no sólo con la importancia que ha adquirido el desarrollo tecnológico para el desarrollo de nuestras sociedades, sino, sobre todo, a los feminismos y los movimientos de liberación de la mujer que se desarrollaron con fuerza a partir de la década anterior ${ }^{2}$. Además, como expo-

1. SCHWARTZ, Ruth. «From Virginia Dare to Virginia Slims: Women and Technology in American Life». Technology and Culture 20, 1 (1979), pp. 51-63. STANLEY, Autumn. «Women Hold Up Two-Thirds of the Sky: Notes for a Revised History of Technology». En P. Hopkins, P (ed.) Sex/Machine. Readings in Culture, Gender and Technology. Indiana, Indiana University Press, 1998, pp. 17-32. TURKLE, Sherry. "Computational reticence. Why women fear the intimate machine». En P. Hopkins, P (ed.) Sex/Machine. Readings in Culture, Gender and Technology. Indiana, Indiana University Press, 1998, pp. 365-380. 2. Wajcman, Judy. El tecnofeminismo. Madrid, Ediciones Cátedra, 2006, pp 25-26.

Feminismo/s 14, diciembre 2009, pp. 163-182 
nen González y Pérez Sedeño ${ }^{3}$, también guarda relación con la investigación y reivindicaciones feministas iniciales orientadas hacia la visibilización del papel jugado por las mujeres en la ciencia y la denuncia de sus condiciones de desigualdad.

Según expone Wajcman ${ }^{4}$, los estudios feministas iniciales de las tecnologías, de talante liberal, celebraban el desarrollo tecnológico y convocaban a las mujeres a participar en ello. Las feministas liberales consideraban que la subrepresentación de las mujeres en las tecnologías se explicaba, básicamente, por la invisibilización del papel de las mujeres en su desarrollo y, así y también, por una imagen masculinizada de las tecnologías, que, de cambiarse, propiciaría el acceso de más mujeres, y así la igualdad entre mujeres y hombres en lo tecnológico ${ }^{5}$. De este modo, la responsabilidad del cambio recaía sobre las mismas mujeres, dejando lo tecnológico prácticamente libre de cuestionamiento.

Pronto se oyeron las críticas de las ecofeministas y las feministas socialistas $^{6}$. A diferencia de las liberales, éstas criticaban el determinismo tecnológico anterior al considerar las tecnologías como parte del sistema patriarcal, cuyo desarrollo tenía efectos negativos para las mujeres ${ }^{7}$. Por un lado, las ecofeministas consideraban a la mujer, a diferencia del hombre, naturalmente cuidadora, pacifista y relacionada con la naturaleza. La tecnología se presentaba de forma antagónica a la naturaleza, y así, a la mujer. Para ellas, pues, la tecnología estaba profundamente masculinizada, fortaleciendo el patriarcado y la opresión de la mujer. Con ello, consiguieron mostrar que la tecnología estaba afectada por las relaciones de género, pero cayeron en el esencialismo y fomentaron la tecnofobia entre las feministas hasta principios de los 90, conllevando así, un profundo distanciamiento entre la tecnología y el género femenino. Por otro lado, los estudios de las feministas socialistas, partiendo de los estudios constructivistas de la tecnología ${ }^{8}$, demostraron la importancia de lo social y el género para explicar el desarrollo tecnológico, y, a diferencia de las

3. GonZÁlez, Marta y Pérez Sedeño, Eulalia. «Ciencia, Tecnología y Género». Revista Iberoamericana de Ciencia, Tecnología, sociedad e innovación, 2 (2002), pp 5:1.

4. WAJCMAN, Judy, Op. cit, pp. 25-30.

5. STANLEY, Autumn. Op. cit, pp.17-19.

6. WAJCMAN, Judy. El tecnofeminismo. Op.cit, pp.30-48; WAJCMAN, Judy. «Feminist Theories of Technology». Cambridge Journal of Economics Advance Access. doi:10.1093/cje/ ben057, (2009), pp. 1-10.

7. COREA, Gena. Man Made Women: How new reproductive technologies affect women. Boston, Routledge, 1985.

8. WAJCMAN, Judy. «Reflections on Gender and Technology Studies: In What State is the Art?» Social Studies of Science, Vol. 30, No. 3 (2000), pp. 447-464. 
ecofeministas, visibilizaron las potencialidades de cambio 9 . De todos modos, como Wajman ${ }^{10}$ reconoce, su insistencia en la reproducción de las jerarquías de género y en la exclusión y discriminación de las mujeres en lo tecnológico, contribuyeron a consolidar el pesimismo de las feministas respecto a las posibilidades emancipadoras que podía conllevar el uso y la práctica tecnológica.

A partir de los años 90, con la irrupción de las postfeministas junto con la impresionante expansión de las nuevas tecnologías de la información y comunicación, entre ellas Internet, volvió el optimismo entre las feministas, que se acompañó de un renacimiento del panorama teórico respecto a sus efectos. Esta vez, el optimismo seguía mostrándose crítico con las desigualdades de género existentes, pero enfatizaba y apostaba por las nuevas y mejores posibilidades de participación para las mujeres a través de las tecnologías, sobre todo las nuevas TIC. Este potencial transformador radicaba en las nuevas posibilidades de apropiación por parte de las mujeres por la novedad de estas tecnologías, sus procesos horizontales de funcionamiento, la generación de nuevos espacios a ocupar como el ciberespacio, y, sobre todo, en las posibilidades que ofrecían para deconstruir categorías prefijadas y unitarias como los mismos géneros. Así pues, Haraway ${ }^{11}$, y buena parte de la literatura cyberfeminista que la sigue ${ }^{12}$, enfatizan el potencial liberador de la ciencia y la tecnología y vuelven a vincular tecnología con progreso, entendido como beneficioso para la emancipación social. Cyberfeministas, postestructuralistas y queer, reivindican una tecnociencia feminista que reconozca sus propias potencialidades y sus propios cementos contingentes y localizados ${ }^{13}$. Para ello

9. Cockburn, Cynthia. Brothers. Male Dominance and Technological Change. Londres, Pluto Press, 1983. Wajcman, Judy. Feminism confronts technology. Pennsylvania, The Pennsylvania State University Press, 1991.

10. WajCMAn, Judy. Reflections on Gender... Op. cit., pp. 449-450.

11. HARAWAY, Donna J. «A Cyborg Manifesto: Science, Technology, and Socialist-Feminism in the Late Twentieth Century», en P. Hopkins (ed), Sex/Machine. Readings in Culture, Gender and Technology. Indiana, Indiana University Press, 1998, pp 434-468.

12. Hawthorne, Susan; Klein, Renate. Cyberfeminism: Connectivity, Critique + Creativity. Australia, Spinifex, 1999; Flanagan, Mary; BoOth, Austin. Reload. Rethinking women + cyberulture. Massachussetts, MIT, 2002; SVENINGSON ELM, Malin; SundEn, Jenny. Cyberfeminism in Northern Lights: Digital Media and Gender in a Nordic Context. Cambridge, Cambridge Scholars Publishing, 2007; Plant, Sadie. Zeros + Ones. Digital women + the new technoculture. Nueva York, Doubleday, 1997.

13. Haraway, Donna. Op. cit.; Plant, Sadie. Op. cit.; Curier, Dianne. «Feminist Technological Futures: Deleuze and Body/Technology Assemblages». Feminist Theory, vol. 4, no 3 (2003), pp. 321-338; FAULKNER, Wendy. «Dualisms, Hierarchies and Gender in Engineering». Social Studies of Science, vol. 30, $\mathrm{n}^{\circ} 5$ (2000), pp. 759-792; LANDSTROM, Catharina. "Queering feminist technology studies». Feminist Theory vol. 8, no 7 (2007), pp. 7-26.

Feminismo/s 14, diciembre 2009, pp. 163-182 
cuestionan, profundamente, las categorías homogéneas, unitarias y fijas que se establecían de lo femenino, masculino y tecnológico. Con ello celebran la hibridación, fluidez, transformación y performatividad del género, pero también de lo tecnológico y de lo que sucede entre ellos.

Entrado el siglo XXI, el optimismo de los 90 se ve atenuado por una realidad que sigue planteando antiguas problemáticas como la subrepresentación de las mujeres en las tecnologías, su invisibilización y discriminación en los entornos TIC, las dificultades de acceso y apropiación de las nuevas TIC por parte de las mujeres, sea como usuarias o como desarrolladoras. Las barreras de acceso siguen siendo significativas para la participación de las mujeres en la toma de decisiones y el diseño de las tecnologías del futuro. En este sentido, las autoras tecnofeministas como Wacjman, herederas de los estudios constructivistas de la ciencia y tecnología, cuestionan el optimismo de los 90 y la efectividad de la deconstrucción del género que enturbia las discriminaciones a las mujeres existentes en las $\mathrm{TIC}^{14}$. Empero, a su vez, refuerzan el carácter fluido, performativo y mútuamente constitutivo del género y las tecnologías que, definitivamente, nos impide hablar estrictamente de los impactos de las tecnologías sobre las mujeres.

Seguidamente pues, y para enfatizar la dimensión performativa y las posibilidades de cambio que conlleva el desarrollo de esta sociedad no nos referiremos a los efectos en tanto a positivos o negativos para las mujeres, sino como retos y potencialidades que requieren ser contextualizadas. De este modo, en el siguiente apartado, con una breve revisión de la literatura al respecto y el análisis de los datos públicos para el Estado Español, presentaremos el principal reto que para las mujeres conlleva el actual desarrollo de la sociedad de la información, esto es, la brecha digital de género. Sin embargo, su estudio, así como la evolución de la sociedad de la información, muestran tendencias de cambio, a corto y medio plazo. Ello se presentará en el último apartado donde las nuevas presencias operadas por las mujeres en el desarrollo tecnológico devienen su mejor potencial para contribuir y sacar provecho del desarrollo de la sociedad de la información.

14. WaJCMAn, Judy. Reflections on Gender...Op. cit.; WajCMAn, Judy. El tecnofeminismo... Op.cit.; WAJCMAN, Judy. Feminist theories...Op. cit. 


\section{La brecha digital de género}

Con la expansión de las TIC, a finales de los años 90, aparece el término brecha digital ${ }^{15}$. Ésta suma varios tipos de exclusión digital e impacta de manera diferente a distintas categorías de la población. La edad, la pertenencia étnica, la categoría socio-profesional o el lugar, o país, de residencia constituyen algunos factores que conllevan una exclusión social agraviada por una exclusión digital.

Las feministas han demostrado, también, la existencia de la brecha digital de género ${ }^{16}$. Si bien el término, en sus inicios, sólo se refería a las diferencias de acceso a las infraestructuras TIC entre mujeres y hombres, actualmente, se ha visto ampliada a la distancia entre sexos de las capacidades de acceso, pero también de uso, apropiación y las posibilidades de participación en el mismo diseño y desarrollo tecnológico ${ }^{17}$.

En este artículo, de forma breve, presentamos el análisis de la brecha digital de género en el Estado Español de forma comparada con las medias Europeas. El análisis se ha realizado a partir de los datos públicos existentes. Se han considerado, por un lado, los datos del INE referentes a la Encuesta sobre equipamiento y uso de tecnologías de la información y comunicación en los hogares y a los Indicadores del sector de tecnologías de la información y las comunicaciones. Por otro lado, y para complementar la ausencia de algunas

15. La atribución del término no es clara. De todos modos, las contribuciones de Norris y Castells consolidaron su importancia y uso a todos los niveles. NORRIS, Pippa. Digital Divide. Civic Engagement. Information Poverty, and the Internet Worldwide. Cambridge, Cambridge University Press, 2001. CASTELLS, Manuel, La galaxia Internet. Reflexiones sobre Internet, empresa y sociedad, Barcelona, Arete, 2001.

16. RASMUSEN, Bente; HAPNES, Tove. «Excluding Women from Technologies of the future? A case Study of the Culture of Computer Science», en P. Hopkins (ed.) Sex/Machine. Readings in Culture, Gender and Technology. Indiana, Indiana University Press, 1998, pp. 381-394; CASTAÑO, Cecilia. La segunda brecha digital. Madrid, Cátedra, 2008; LifF, Sonia y SHEPHERD, Adrian. "An evolving gender digital divide?». Oxford Internet Institute. Internet Issue Brief. no 2 (2004), pp 1-10; KenNEDy, Tracy; Wellman, Barry y KLEMENT, Kristine. «Gendering the digital divide». It and Sociedy. vol. 1, $\mathrm{n}^{\circ} 5$ (2003), pp. 72-96.

17. CAStaño, Cecilia. La segunda brecha... Op. cit. Castaño, Cecilia. Las mujeres y las tecnologías de la información. Internet y la trama de nuestra vida. Madrid, Alianza Editorial, 2005; PÉREZ SAlAZAR, Gabriel. «El estado del arte de la brecha digital», en D. Crovi (coord.) Hacia la sociedad de la información y el conocimiento. México, FCPy UNAM, 2004, pp. 137-145; SAINZ, Milagros; CASTAÑO, Cecilia y ARTAL, Margarita. Anàlisi del concepte d'alfabetització digital i de les seves implicacions en l'estudi de la divisió digital del gènere. IN3.WP08-001. 2008. <http://www.uoc.edu/in3/dt/cat/sainz-castanoartal.html>, consultado el 15-10-2009.

Feminismo/s 14, diciembre 2009, pp. 163-182 
informaciones, se han utilizado informes de investigación como los realizados por distintas universidades o la Unión Europea ${ }^{18}$.

Los datos públicos oficiales, aunque crecientes, siguen siendo muy limitados para estudiar a fondo la brecha digital de género. Por un lado, se muestran de forma poco lineal en el tiempo, de modo que aparecen y desaparecen indicadores desagregados por sexos según el año. Por otro lado, no se permiten cruces variados entre sexos y el resto de variables. Además de ello, el género suele tratarse de forma genérica sin contemplar su diversidad y haciendo sólo referencia a la segregación por sexos. Finalmente, los datos disponibles no permiten ahondar en aspectos más específicos de usos intensivos, motivaciones y otras variables más allá de los usos comerciales de las TIC.

\subsection{Equipamientos e infraestructuras}

Para entender el fenómeno de la brecha digital entre mujeres y hombres en España, cabe introducir la realidad del contexto español en cuanto a equipamientos e infraestructuras. España tiene demasiadas asignaturas pendientes para facilitar el desarrollo de la sociedad de la información de forma adecuada. Aunque los equipamientos e infraestructuras TIC van en aumento, los datos siguen mostrando que existen importantes deficiencias y que su mejora sigue siendo lenta y distante respecto a otros países europeos.

En el conjunto del Estado, y con datos del $2008^{19}$, sólo un $63,6 \%$ de los hogares disponen de ordenador. Esto representa un fuerte incremento respecto al año 2003 (43,3\%), pero sólo un punto porcentual más respecto el año anterior. Por Comunidades Autónomas los grados más elevados de tenencia de ordenador se observan en Madrid (71,9\%), Catalunya (71\%), Euskadi $(66,4 \%)$ y Cantabria $(66,2 \%)$. Los porcentajes menos elevados se presentan

18. FÉlIX, Bernard. «Employment and earnings in high-tech sectors». Statistics in Focus, $\mathrm{n}^{\circ} 32(2007)<\mathrm{http} / / /$ www.cec-wys.org/docs/Empl\%20and\%20earnings.pdf>, consultado el 15 de octubre de 2009; LA SALLE e ICSA. Estudi DonaTic:Una primera reflexió sobre la dona tecnologica. Barcelona, $2007<\mathrm{http} / / / \mathrm{www}$.salle.url.edu/donaTIC/index. html>, consultado el 15-10-2009. MERI, Thomas. «Highly qualified workers in science and technology». Statistics in focus, $n^{\circ} 103$ (2007) <http://epp.eurostat.ec. europa eu/cache/ity_offpub/ks-sf-07-103/en/ks-sf-07-103-en.pdf>, consultado el 16-10-2009. WiLLEN, Hakan. «Measuring gender differences among Europe's knowledge workers». Statistics in Focus n ${ }^{\circ} 12$ (2006), <http://epp.eurostat.ec.europa.eu/cache/ity_offpub/ksns-06-012/en/ks-ns-06-012-en.pdf>, consultado el 16-10-2009.

19. INE. Encuesta sobre Equipamiento y Uso de Tecnologías de la Información y Comunicación en los hogares. Datos disponibles en: <http://www.ine.es/jaxi/menu. do? type $=$ pcaxis\&path $=/ \mathrm{t} 25 / \mathrm{p} 450 \&$ file $=$ inebase $\& \mathrm{~L}=0>$, consultado el 16-10-2009. 
en Extremadura (53,3\%), Galicia (53,6\%), Castilla la Mancha (53,5\%), Ceuta (56,8\%) y Murcia (57,2\%).

En cuanto a la disposición de Internet, los porcentajes se reducen y, en el 2008, sólo el 51\% de los hogares del Estado Español tienen acceso a Internet. Aunque esta cifra ha sido creciente desde el 2004, cuando se encontraba en un $33,6 \%$, sigue siendo muy inferior a la media Europea. Por Comunidades Autónomas, las que presentan mayores porcentajes de acceso a Internet son Madrid (62,3\%) y Catalunya (60,1\%). Illes Balears, Euskadi, Cantabria, Navarra, Aragón, Asturias, La Rioja y Canarias presentan tasas superiores al 50\%, pero Galicia (39,7\%), Castilla la Mancha (40,5\%), Castilla León (41,7\%), Murcia $(42,3 \%)$, Extremadura (42,9\%) y Andalucía (43,7\%) presentan cifras muy inferiores a la media. En cuanto al tipo de conexión, menos de la mitad de las viviendas que se conectan a Internet $(44,6 \%)$ lo hacen por banda ancha ${ }^{20}$.

Con respecto a los equipamientos en tecnologías en el ámbito escolar, para el curso 2006-2007, había cerca de 7 alumnos/as por ordenador. La situación en los centros privados es peor, pues muestra una cifra de 10 alumnos/ as por ordenador mientras que en los públicos no llega a 6 alumnos/as por ordenador. Desde el 2000, la evolución ha sido lenta, pero ha ido mejorando. Destaca el esfuerzo que han llevado a cabo Comunidades Autónomas como Extremadura donde sólo hay 2,3 alumnos/as por ordenador o Euskadi con una cifra de 5. Sin embargo, Illes Balears, Madrid, Valencia, Melilla y Canarias destacan por carecer de ordenadores, con cifras iguales o superiores a 9 alumnos/as por ordenador ${ }^{21}$

En enero del 2008, el 95\% de las empresas del Estado Español de 10 asalariados/as o más disponían de conexión a Internet, datos que han aumentado, aproximadamente, 13 puntos desde el 2003. Por Comunidades Autónomas, las empresas con sede en Madrid, Catalunya, Euskadi y Navarra presentan una mayor intensidad en el uso de las TIC. Las empresas de Castilla la Mancha, Extremadura, Ceuta y Melilla y Cantabria presentan las menores intensidades. Respecto al resto de países europeos, el Estado Español se sitúa ligeramente por encima de la media EU-25 (94\%) respecto a las empresas en cuanto al acceso a Internet y la disposición de ordenador. Sin embargo, en otros aspectos, como la tenencia de web, se sitúa muy por debajo de la media EU-25 (66\%), ya que sólo un 54\% de las empresas disponen de ella. Con respecto a las compras y ventas a través del comercio electrónico, el Estado Español

20. Ibíd.

21. INE. Tecnología de la información en la enseñanza no universitaria. Datos disponibles en: $<$ http://www.ine.es/jaxi/menu.do?type=pcaxis\&path=/t13/p022\&file=inebase $\& \mathrm{~L}=0>$, consultado el 16-10-2009. 
(19\% y $10 \%$ respectivamente) muestra unas cifras que se sitúan aproximadamente en la mitad de la media EU-25 (30\% y 19\% respectivamente) ${ }^{22}$.

\subsection{Brecha digital de género en los usos y prácticas con ordenador}

Una vez más, el análisis de los datos para usos y prácticas tecnológicas muestra una realidad muy mejorable y, por lo tanto, lejana de otros países europeos. En España, el perfil de persona usuaria del ordenador e Internet sigue siendo hombre, blanco, joven, con estudios universitarios y habitante de una gran ciudad. Por lo tanto, además del género se observan importantes disparidades por edad, nivel de estudios y lugar de residencia.

Las mujeres acceden al ordenador y a Internet en menor medida que los hombres. Para las personas que lo han utilizado alguna vez, la distancia entre sexos supera los 7 puntos porcentuales. A medida que aumenta la frecuencia de uso, disminuye el porcentaje de mujeres. En la frecuencia regular, al menos una vez por semana, la distancia se sitúa cerca de los 9 puntos porcentuales ${ }^{23}$. Además, para el año 2007, la frecuencia regular de uso de las mujeres de España (40\%) es muy inferior a la media Europea, EU-25 (48\%) y EU-15 (50\%), aunque la brecha digital de género en España también es muy similar a las medias Europeas ${ }^{24}$.

El tipo de tareas y usos particulares que se realizan de Internet y con el ordenador también muestra diferencias importantes entre mujeres y hombres. En este sentido, vemos una reproducción de la división sexual del trabajo en los usos de Internet. Ellas se preocupan más de la salud y las vacaciones familiares, ellos de consultar los medios de comunicación y descargas de software. Además, se aprecia una menor dedicación al ocio por parte de las mujeres, unos 10 puntos porcentuales por debajo de los hombres y casi 20 en el caso de los juegos ${ }^{25}$. De forma similar, en casi todas las tareas informáticas los hombres presentan porcentajes superiores a las mujeres, menos en el caso de tareas relacionadas con documentación. Sobre todo, destaca la enorme distan-

22. INE. Encuesta sobre el uso de TIC y comercio electrónico en las empresas. Datos disponibles en: http://www.ine.es/jaxi/menu.do?L=0\&type=pcaxis\&path=/t09/e02\&file=inebase, consultado el 16-10-2009.

23. INE. Encuesta sobre Equipamiento y Uso de Tecnologías... Op. cit.

24. INE. Nuevas tecnologías de la información y la comunicación. Datos Europeos. Datos disponibles en: http://www.ine.es/jaxi/menu.do?type=pcaxis\&path=/t25/p450/ e01/\&file=pcaxis, consultado el 19-10-2009.

25. INE. Encuesta sobre Equipamiento y Uso de Tecnologías... Op. cit. 
cia entre sexos, hasta 20 puntos porcentuales, de las tareas de mantenimiento, programación o instalación, que sobre todo las realizan los hombres ${ }^{26}$.

\subsection{Brecha digital de género en el conocimiento avanzado y en la participación en los sectores TIC}

Sin duda, la mayor brecha digital de género se observa en el acceso al conocimiento avanzado del ordenador, Internet y sus aplicaciones, esto es, a través de los estudios y empleos TIC. La subrepresentación de las mujeres en los estudios tradicionalmente tecnológicos es evidente tanto en la formación profesional como en la educación superior. Aunque ha crecido en los últimos años, recientemente parece haberse estancado y la situación empieza a empeorar para las mujeres. Esta tendencia parece ser compartida con la mayoría de países occidentales.

Según los datos del curso 2006-2007 las mujeres sólo representan cerca del 17\% de estudiantes de formación profesional en informática y, en el bachillerato, una vez más, sólo aproximadamente un 20\% de las estudiantes cursa el itinerario tecnológico ${ }^{27}$. Para los estudios universitarios en ingenierías la situación es muy similar. En las carreras de ingeniería técnica las mujeres representan un 25\% del alumnado, con porcentajes bastante menores en el caso de las telecomunicaciones e informática. En las ingenierías superiores la situación parece mejorar, así, en general, representan, aproximadamente, un $31 \%$ del alumnado (para telecomunicaciones en torno a un 26\% y para informática un 17\%). A nivel de tercer ciclo la situación es similar a los estudios superiores. Las mujeres representan un $28 \%$ de las personas que cursan estudios de doctorado en el área de ingeniería y tecnología. De todos modos, y una vez más, los porcentajes disminuyen en el caso de las mujeres matriculadas en doctorados en Informática (18,35\%) y Telecomunicaciones (18,36\%) $\mathrm{y}$, de forma preocupante, muestran una tendencia de estancamiento o incluso descenso con el tiempo ${ }^{28}$.

En los sectores laborales TIC, las mujeres también se encuentran subrepresentadas. Según datos de Eurostat, las mujeres representan alrededor de un $28 \%$ de las personas empleadas en estos sectores. Además, la segregación horizontal y vertical también afecta los sectores TIC. La gran ausencia de mujeres se encuentra en tareas de mantenimiento, pero además, debido a la

26. Ibíd.

27. INE. Enseñanzas anteriores a la universidad. Datos disponibles en: <http://www.ine.es/jaxi/ menu.do? type=pcaxis\&path=/t13/p001\&file=inebase $\& \mathrm{~L}=0>$, consultado el 16-10-2009.

28. INE. Estadística de Enseñanza Universitaria. Datos disponibles en: http://www.ine.es/jaxi/ menu.do?type=pcaxis\&path=/t13/p405\&file=inebase $\& \mathrm{~L}=0$, consultado el 16-10-2009.

Feminismo/s 14, diciembre 2009, pp. 163-182 
segregación vertical, se encuentran en porcentajes muy inferiores en los puestos directivos. En todos los países europeos las mujeres están peor pagadas que los hombres, pero el Estado Español presenta una de las distancias salariales más elevadas de Europa ${ }^{29}$.

Así pues, como sumario, en el Estado Español hay mucho por recorrer en materia de brecha digital de género y así, facilitar el acceso, uso, apropiación y participación igualitaria de las TIC entre sexos. La disponibilidad de equipamientos e infraestructuras aún no asegura la igualdad de oportunidades entre territorios y grupos poblacionales, afectando así, a las mujeres. Ellas presentan porcentajes de uso del ordenador e Internet por debajo de los hombres, sobre todo en variedad de usos y en los usos más frecuentes. La brecha digital de género es mucho más preocupante si atendemos a la cuestión del acceso al conocimiento avanzado y a las posibilidades de participación en el desarrollo de las tecnologías y la sociedad de la información. Las mujeres representan menos del 30\% de estudiantes de ingeniería y de trabajadoras TIC. Además, y una vez más, los estudios y sectores laborales TIC se ven afectados por una segregación horizontal y vertical que perjudica a las mujeres, pues ellas se ven imposibilitadas al acceso a los puestos de mayor responsabilidad, salario y prestigio. En este aspecto, la situación aunque mejoró respecto el pasado, parece haberse estancado y puede retroceder.

\section{Las nuevas presencias de mujeres en la sociedad de la información}

\subsection{Nuevas presencias y diversidad de perfiles de acceso a los trabajos TIC}

A pesar de la permanencia de la brecha digital de género, las mismas fuentes de datos, nueva literatura y los resultados de la investigación de Donestech ${ }^{30}$ señalan importantes posibilidades de cambio para el futuro, pues nuevas presencias de mujeres en las tecnologías aparecen y se visibilizan. En este sentido, los datos oficiales señalan que la brecha digital de género de acceso a las TIC se reduce, e incluso se invierte, en las edades más jóvenes. En cuanto a las posibilidades de apropiación tecnológica por parte de las mujeres y sus posibilidades de participación en el desarrollo de las tecnologías y en la sociedad de la información, aunque no parece que la brecha se supere, existen, también, importantes elementos que apuntan hacia cambios en el futuro.

29. Willen, Hakan. Op. cit.; Meri, Thomas, Op cit.; FÉlix, Bernard. Op. cit.; La SAlle e ICSA, Op. cit.

30. Disponible en: <http://www.Donestech.net>, consultado el 16-10-2009. 
Según los datos del INE ${ }^{31}$, en las edades más jóvenes las mujeres presentan unos mayores porcentajes de uso del ordenador e Internet que los hombres. Por otra parte, porcentajes de uso muy superiores a la media de población. De seguir esta tendencia la brecha digital de género puede desaparecer, al menos en cuanto al acceso y uso básico de las TIC. Esta pauta, además, parece repetirse en la mayoría de países occidentales.

Aunque en menor grado y de forma menos evidente que los datos expuestos, algunos estudios también apuntan a cambios en cuanto a la formación y la participación en el mundo laboral TIC. Este hecho es importante pues implica mayores posibilidades de participación de las mujeres en el desarrollo tecnológico y de la sociedad de la información futura. En este sentido, aunque los porcentajes de estudiantes y trabajadoras TIC sean muy reducidos en los países occidentales, algunos estudios en países asiáticos o en Sudáfrica, muestran que las estudiantes y trabajadoras TIC, representan porcentajes prácticamente paritarios ${ }^{32}$.

Pero, más allá de los estudios tradicionalmente considerados TIC, como Informática y Telecomunicaciones, existe otra gran tendencia de cambio. El desarrollo de la sociedad de la información impregna y se alimenta de una gran diversidad de disciplinas y áreas de conocimiento que, antiguamente, se mostraban alejadas de lo tecnológico como la biblioteconomía, la comunicación, la economía, la biología o el arte ${ }^{33}$. En estos campos, la presencia de mujeres es mayoritaria y los usos que realizan de las tecnologías cada vez son más avanzados. De esta forma, las mujeres, como usuarias avanzadas de las tecnologías, pueden generar nuevas demandas y formas de uso, influenciando, a su vez, la creación y evolución de artefactos y aplicaciones tecnológicas. Además, muchas de estas mujeres se incorporan en profesiones TIC y adquieren nuevas capacidades de decisión en el desarrollo tecnológico futuro.

31. INE. Encuesta sobre Equipamiento y... 2008. Op. cit.

32. GALPIN, Vashti. «Women in Computing around the World». SIGSE Butlletin. Vol 32, ${ }^{\circ}$ 2 (2002), pp. 94-106. WAJCMAn, Judy y PнAм LoвB, Le Ahn «The Gender Relations of Software Work in Vietnam». Gender Technology and Development. Vol. 11, nº 1 (2007), pp 1-26.

33. Alsina, Pau. Arte, ciencia y tecnología. Barcelona, UOC, 2007; CuCKIER, Wendy; SHORTT, Denise y DEVINE, Irene. «Gender and Information Technology: Implications of Definitions». Journal of Information Systems Education. Vol. 13, $\mathrm{n}^{\circ} 1$ (2002), pp.715; JESSE, Jolene K. «The Poverty of the Pipeline Metaphor: The AAAS/CPST Study of Nontraditional Pathways into IC/CS Education and the Workforce». En J. M. Cohoon y W. Aspray (Eds) Women and Information Technology: Research on Under-Representation. Massachussets, MIT Press. 2006, pp 239-278.

Feminismo/s 14, diciembre 2009, pp. 163-182 
De hecho, autores como Bartol y Aspray ${ }^{34}$ exponen que un importante número de trabajadores/as TIC llegan a ello por caminos diferentes a los estudios tradicionalmente considerados TIC. De forma similar, Faulkner exponía que en el Reino Unido aproximadamente un $62 \%$ de los/as graduados/as que trabajan en puestos TIC no procedían de este tipo de estudios ${ }^{35}$. Indagando en esta cuestión, Jesse ${ }^{36}$ afirma que el camino al estudio o trabajo no es lineal, tampoco en lo tecnológico, pues no únicamente se requiere formación reglada en informática para trabajar en su desarrollo. De acuerdo con ello y en relación a las mujeres, Chapple ${ }^{37}$ muestra que muchas mujeres están desarrollando trabajos TIC y que los cursos cortos y la formación laboral en ONG son vías no tradicionales que permiten a más mujeres entrar en el mundo laboral TIC.

Como insistió la literatura y práctica cybefeminista ${ }^{38}$, las características del desarrollo actual de la sociedad de la información, con un importante componente comunicacional y de trabajo horizontal y en red, otorgan mayor relevancia a las aptitudes consideradas femeninas. Un ejemplo de esta corriente, es el trabajo y el Manifiesto de la Zorra Mutante del colectivo cyberfeminista VNS Matrix ${ }^{39}$, que desde la ironía y la parodia ocupan la red generando nuevos modelos de participación. Además, la expansión de la web 2.0 es testimonio del auge de las webs de redes sociales, que, según los recientes estudios, convierten a las TIC en propio de lo cotidiano y estimulan la participación intensiva de las mujeres. De hecho, para algunas aplicaciones la presencia de mujeres es mayoritaria, como en el caso de los weblogs ${ }^{40}$.

34. BARTOL, Kathryn, M; Aspray, William. «The Transition of Women from the Academic World to the IT Workplace: A Review of the Relevant Research». En J. M. Cohoon, y W. Aspray (Eds.) Women and Information Technology: Research on Under-Representation. Massachusetts, MIT Press, 2006, pp. 377-420.

35. FAULKNER, Wendy. Women, gender in/and ICT: Evidence and reflections from the UK. SIGIS. D02-Part3, 2002. <http://www.rcss.ed.ac.uk/sigis/public/displaydoc/full/D02_ Part3>, consultado el 16-10-2009, p. 8

36. JESSE, Jolene K. Op. cit.

37. Chapple, Karen. «Foot in the Door, Mouse in Hand: Low-Income, Women, Short-Term Job Training Programs, and IT Careers» en J. M. Cohoon y W. Aspray (Eds.) Women and Information Technology: Research on Under-Representation. Massachussets, MIT Press, 2006, pp. 439-470.

38. Sveningson Elm, Malin y Sunden, Jenny. Op. cit.; Plant, Sadie. Op. cit. Chapple, Karen. Op. cit. En relación al cyberfeminismo práctico ver los trabajos de Old Boys Network o los manifiestos de VNS Matrix < http://www.obn.org>, consultado el 10-06-2009.

39. Disponible en: <http://www.sysx.org/gashgirl/vns/text/pinkmani.htm>, consultado el 16-10-2009.

40. CaRSTEnSEN, Tanja. «Gender Trouble in Web 2.0. Gender perspectives on social network sites, wikis and weblogs». Online Proceedings of the 5th European Symposium on 
De este modo, se vislumbra que habrá cambios de futuro porque las niñas y las mujeres jóvenes están accediendo más que los hombres a las TIC, pero, sobre todo, porque las mujeres están accediendo, de forma creciente, a los trabajos TIC a través de otras vías y disciplinas de las antiguamente consideradas tecnológicas. Ello, no sólo puede incrementar el número de mujeres en las tecnologías, sino, sobre todo, implicar nuevas formas de trabajar y desarrollar la sociedad de la información futura. En este sentido, corroborando y ampliando las posibilidades de cambio se presentan, a continuación, los principales resultados de la investigación Donestech al respecto.

\subsection{De Donestech: la diversidad disciplinar en el acceso y la práctica tecnológica.}

En el 2006, tres mujeres provenientes de la práctica tecnoactivista y con experiencia en la investigación social iniciaron una investigación para conocer cómo las mujeres que ya estaban participando de lo tecnológico habían accedido a las tecnologías, los usos que hacían de ellas y cuáles eran sus deseos e inquietudes respecto a su práctica tecnológica y a la sociedad de la información en general. Así nació en Barcelona el colectivo Donestech y el proyecto Código Lela que, con el tiempo, se han expandido en número y diversidad de personas participantes, formatos, actividades, lugares y experimentaciones ${ }^{41}$.

El colectivo Donestech inició sus actividades a través de la investigación sobre los accesos, usos y deseos de las mujeres tecnólogas persiguiendo los objetivos de visibilizar sus experiencias y especificidades, potenciar el acceso de más mujeres en las tecnologías, y seguir enredándose e incorporando las tecnologías en la investigación social y en las propias prácticas activistas ${ }^{42}$. Para ello, se creó una web en software libre, se recopiló información sobre mujeres y tecnologías, se generaron, y se participó, en encuentros y talleres y, sobre todo, se recopilaron testimonios y trayectorias de mujeres tecnólogas.

La investigación de Donestech se propuso no partir de una definición predeterminada de mujer y de tecnología para, así, no iniciarse en un universo predefinido y específico de mujeres tecnólogas. De esta forma, el estudio partió de un sentido amplio de la palabra y no prefijó los sujetos de análisis. Con ello, incluyó

Gender and ICT. 2009. <http://www.informatik.uni-bremen.de/soteg/gict2009/proceedings/GICT2009_Carstensen.pdf> , consultado el 10-06-2009.

41. Para más información sobre Donestech consultar la web: <http://www.Donestech.net>.

42. La investigación se inició para Catalunya con la ayuda de investigación 2006-2007 del Institut Català de les Dones de la Generalitat de Catalunya. Pronto se amplió al Estado Español con la ayuda del Plan Avanza 2006 del Ministerio de Industria, Turismo y Comercio.

Feminismo/s 14, diciembre 2009, pp. 163-182 
a todas las mujeres que se identificaron como tecnólogas, como mujeres que participan de las tecnologías de forma avanzada, fuese por su práctica tecnológica, por los ámbitos donde la desarrollan, sean remunerados o no remunerados, de placer, de información, de producción, etc. Teniendo esto en cuenta, se hizo una llamada a través de Internet para responder un cuestionario semiabierto on-line. Su difusión se realizó en el 2007 a partir de mujeres tecnólogas procedentes de ámbitos distintos, como el alumnado de informática de la UOC, asociaciones de mujeres empresarias y ejecutivas, colectivos de mediactivistas de Barcelona y organizaciones y redes de género, simulando así la técnica de la bola de nieve en un contexto virtual. También se difundió el proyecto y la posibilidad de responder el cuestionario en eventos especializados en mujeres y/o en tecnología además de enviar la propuesta a distintas listas de correo especializadas en tecnología y/o género invitando a su difusión. El cuestionario fue contestado por 302 tecnólogas de diferentes nacionalidades, y, además, se realizaron cerca de 60 entrevistas en profundidad y 3 grupos de discusión, en diferentes Comunidades Autónomas.

El perfil más común de mujeres tecnólogas que respondieron al cuestionario fueron mujeres que mayoritariamente vivían en ciudades de más de 100.000 habitantes, de una edad media de 35 años y que tuvieron la primera experiencia con las tecnologías antes de los 20 años. La mayoría se encontraba en situación activa y ocupada y, en su gran mayoría, tenían estudios universitarios.

Uno de los principales resultados de esta investigación lo constituye la constatación de la enorme diversidad y heterogeneidad presente entre las mujeres tecnólogas, tanto de procedencias disciplinares como de prácticas tecnológicas. Algo más de la mitad de las tecnólogas que respondieron el cuestionario, así como las entrevistadas en profundidad, no han cursado estudios científico-técnicos, aunque mayoritariamente tienen estudios universitarios. Además, entre los tipos de estudios científico-técnicos cursados también se observa una gran variedad disciplinar, desde la informática, a las matemáticas, la arquitectura o la química. Las tecnólogas que no han cursado estudios científico-técnicos son las que más han realizado diversos cursos de capacitación en TIC, tanto a nivel formal como informal, y presentan caminos de aprendizaje tecnológico raramente mencionados y explorados en la literatura sobre participación de las mujeres en las tecnologías. De este modo, se visibilizan las vías de aprendizaje y capacitación tecnológica no lineales, normalmente sobrepuestas, y también las informales, a través de cursos, talleres en centros sociales y, sobre todo, una elevada dosis de trabajo autodidacta a través de Internet, manuales y amistades. En este sentido, una tecnóloga artística y 
programadora de aplicaciones libres de Barcelona expresaba de la siguiente forma su capacitación y entrada en el sector TIC:

Un poco autoinsistente, pinceladas autodidactas, he hecho algún curso, pero no tengo formación sólida de base. Mucho gracias a poder comunicarme y ayudarnos con gente en una situación más o menos similar. En casa tengo ayuda, mi madre es informática y trabaja de analista, y es linuxera. Los cursos, en la medida de lo posible eran subvencionados (del paro o de reciclaje) o, si no, con ayuda de casa. A base de tiempo de contacto, inevitablemente, vas aprendiendo, o sea que, sobre la marcha (Dalzat, 1980) ${ }^{43}$.

Las mujeres tecnólogas tienen un perfil laboral variado y múltiple. Las tecnólogas que contestaron el cuestionario, mayoritariamente, declararon ser pluriempleadas ${ }^{44}$, algunas veces definiendo el ser madre como un trabajo más. Aproximadamente, un 22,8\% afirma tener una ocupación principal del ámbito técnico-social (administrativa, investigadora social, periodista, contable, etc.), un 19,5\% dice tener una ocupación del ámbito científico-técnico (química, analista, programadora, etc.). Un 16,3\%, además, afirma ser estudiante sin especificar en qué campo. Finalmente, cabe destacar que un 14,3\% dice trabajar en el ámbito técnico-cultural-artístico (que responde a ocupaciones como productora audiovisual, diseñadora gráfica, técnica de sonido, etc.). Finalmente, en menores porcentajes, se encuentran los empleos del ámbito técnico-formativo, tanto en secundaria como a nivel universitario. En menor medida, menos de un 5\% cada una, se dedican a actividades técnicopersonales (blog personal, web propia), técnico-domésticas (tareas del hogar, ser madre) y tecno-activistas (como el mediactivismo, activismo social). También, la variabilidad contractual entre las tecnólogas es bastante acusada, desde becarias hasta freelance o funcionarias, aunque casi la mitad tiene contrato laboral y su situación laboral no parece depender de haber cursado o no estudios técnicos.

Las tecnólogas hacen un uso extenso y heterogéneo de las tecnologías. Las TIC, más allá de ser una herramienta de trabajo, forman parte de su vida cotidiana como herramientas de comunicación, gestión, cooperación, entretenimiento, ocio, información y, también, como herramientas de expresión y participación social y política. De este modo lo expresaba una de les Donestech participantes en el proyecto:

43. Para preservar el anonimato se han cambiado las vocales del nombre de la tecnóloga. El año se refiere a su fecha de nacimiento.

44. Entre 2 y 3 trabajos a la vez. Cabe tener en cuenta que se incluyen los trabajos no remunerados, el voluntariado y las tareas familiares o del hogar.

Feminismo/s 14, diciembre 2009, pp. 163-182 
Usuaria: universidad, consultas bancarias, ocio, búsqueda de información...

Como desarrolladora: desarrollo web de venta de entradas servicticket (Aspa.

1976).

Aunque todas las mujeres tecnólogas que contestaron la encuesta son usuarias avanzadas de las tecnologías, casi la mitad participan en la creación o desarrollo de programas, aplicaciones o herramientas TIC. Éstas últimas, además, tienden a realizar menos trabajo en solitario y a utilizar mucho más el software libre. Cerca de la mitad de las mujeres que respondieron, utilizan para algunas tareas también el software libre, aunque el hardware que utilizan es sobre todo propietario. La lista de artefactos, objetos, lenguajes, programas, aplicaciones, dispositivos etc. sería interminable, pero, una vez más, implica el trabajo en, prácticamente, cualquiera de las áreas de actividad humana, desde la experimentación en hardware, a la creación artística, la gestión de bases de datos, la investigación social, la enseñanza secundaria, lo lúdico o la fotografía digital, además y a menudo, de forma superpuesta.

Finalmente, cabe destacar que las tecnólogas participantes en el proyecto de Donestech utilizan y desarrollan las tecnologías de forma crítica y con deseos de cambio para el futuro. En este sentido, apuestan por una mayor expansión e implantación del software libre, por ambientes y vías de aprendizaje amigables para las mujeres, por un desarrollo más sostenible y socialmente justo, por una mejora y ampliación de las posibilidades de transmisión e intercambio de conocimientos y, sobre todo, desean que se acabe con la discriminación de las mujeres y la imagen de que las mujeres no están, o que no son aptas, para las tecnologías, porque, también a ellas, les sigue perjudicando.

\section{Conclusiones}

Aunque la brecha digital de género persiste, su análisis en el contexto del Estado Español y por grupos de edad, apunta a cambios importantes. Por un lado, las niñas y las mujeres jóvenes están accediendo en mayor medida que los hombres a las TIC. Por otro lado, aunque las mujeres son minoría en los estudios y trabajos TIC, la literatura internacional apunta hacia un aumento de su participación en el empleo TIC a través de vías no tradicionales. Las mujeres también se inician en los trabajos TIC a partir del estudio de otras disciplinas diferentes a la informática y las telecomunicaciones y se capacitan en materia tecnológica a través de sus estudios y otras prácticas de aprendizaje, formales e informales, que se superponen.

La investigación de Donestech apunta a conclusiones similares. Las mujeres tecnólogas provienen de una gran diversidad de disciplinas, y, más aún, sus prácticas tecnológicas son diversas y se llevan a cabo en diversos ámbitos. 
Si tenemos en cuenta que estas mujeres participan en las TIC de forma crítica y con voluntad de cambio, el efecto de su presencia en los trabajos TIC puede implicar nuevos cambios en el desarrollo de la sociedad de la información del futuro.

Estas nuevas presencias pueden reforzar el carácter mutuamente constitutivo de las tecnologías y los géneros y, a su vez, su relación fluida y preformativa. Una mayor variedad de géneros y disciplinas participando en el desarrollo tecnológico y de la sociedad de la información puede acabar con la masculinización de lo tecnológico y posibilitar un desarrollo de la sociedad de la información más acorde con la diversidad de géneros y tecnologías existentes y en desarrollo.

La presencia y visibilización de estas mujeres muestra la complejidad de la evolución de la brecha digital de género, pero sobre todo, cuestiona las estructuras sociales de esta sociedad y la forma en que se desarrolla la sociedad de la información. En este sentido, un mayor esfuerzo de investigación de estas presencias es necesario para su visibilización y para ahondar en las condiciones de su participación, y así, asegurar su contribución a atenuar la imagen y práctica tecnológica masculinizada y generar nuevas oportunidades para las mujeres y el destino de la sociedad de la información en su conjunto.

Apunte final:

El 8 de marzo nos viene estupendamente para que se acuerden de nosotras, somos mujeres y nos dejan hacer lo que queramos, un día que de repente lo que parece que no hay durante todo el año, de repente hay un montón de mujeres que hacen... (Colectivo de mujeres dj's. Barcelona)

Si lo fuera cada día...

\section{Bibliografía}

Alsina, Pau. Arte, ciencia y tecnología. Barcelona, UOC, 2007.

BARTOL, Kathryn, M; AsPraY, William. «The Transition of Women from the Academic World to the IT Workplace: A Review of the Relevant Research». En J. M. Cohoon, y W. Aspray (Eds.). Women and Information Technology: Research on Under-Representation. Massachusetts MIT Press, 2006, pp 377-420.

CARSTEnSEN, Tanja. «Gender Trouble in Web 2.0. Gender perspectives on social network sites, wikis and weblogs». Online Proceedings of the 5th European Symposium on Gender and ICT. 2009. <http://www.informatik.uni-bremen. de/soteg/gict2009/proceedings/GICT2009_Carstensen.pdf>, consultado el 10-06-2009.

CASTAÑO, Cecilia. Las mujeres y las tecnologías de la información. Internet y la trama de nuestra vida. Madrid, Alianza Editorial, 2005. 
CASTAÑO, Cecilia. La segunda brecha digital. Madrid, Cátedra, 2008.

CASTELLS, Manuel. La galaxia Internet. Reflexiones sobre Internet, empresa y sociedad, Barcelona, Arete, 2001.

CHAPPLE, Karen. «Foot in the Door, Mouse in Hand: Low-Income, Women, ShortTerm Job Training Programs, and IT Careers» en J. M. Cohoon y W. Aspray (Eds.) Women and Information Technology: Research on Under-Representation. Massachussets, MIT Press, 2006, pp 439-470.

CockBurn, Cynthia. Brothers. Male Dominance and Technological Change. Londres, Pluto Press, 1983.

COREA, Gena. Man Made Women: How new reproductive technologies affect women. Boston, Routledge, 1985.

CuCKIER, Wendy; SHORTt, Denise y Devine, Irene. «Gender and Information Technology: Implications of Definitions». Journal of Information Systems Education. Vol. 13, nº 1 (2002), pp.7-15.

Curier, Dianne. «Feminist Technological Futures: Deleuze and Body/Technology Assemblages». Feminist Theory. Vol. 4, no 3 (2003), pp. 321-338.

FAUlKner, Wendy. «Dualisms, Hierarchies and Gender in Engineering». Social Studies of Science, vol. 30, no 5 (2000), pp. 759-792.

- Women, gender in/and ICT: Evidence and reflections from the UK. SIGIS. D02Part3, 2002. <http://www.rcss.ed.ac.uk/sigis/public/displaydoc/full/D02_Part3>, consultado el 16-10-2009.

FÉLIX, Bernard. «Employment and earnings in high-tech sectors». Statistics in Focus, Science and Tecnology, $\mathrm{n}^{\mathrm{o}} 32$ (2007). <http://www.cec-wys.org/docs/ Empl\%20 and\%20earnings.pdf>, consultado el 15-10-2009.

FLANAGAN, Mary; BOOTH, Austin. Reload. Rethinking women + cyberulture. Massachussetts, MIT, 2002.

GaLPIN, Vashti. «Women in Computing around the World». SIGSE Butlletin. Vol 32, no 2 (2002), pp. 94-106.

GonzÁlez, Marta. PÉrez Sedeño, Eulalia. «Ciencia, Tecnología y Género». Revista Iberoamericana de Ciencia, Tecnología, sociedad e innovación, no 2 (2002), p. 5.

HaraWAY, Donna J. «A Cyborg Manifesto: Science, Technology, and SocialistFeminism in the Late Twentieth Century», en P. Hopkins (ed), Sex/Machine. Readings in Culture, Gender and Technology. Indiana, Indiana University Press, 1998, pp 434-468.

HaWthorne, Susan; Klein, Renate. Cyberfeminism: Connectivity, Critique + Creativity. Australia, Spinifex, 1999.

JESSE, Jolene K. «The Poverty of the Pipeline Metaphor: The AAAS/CPST Study of Nontraditional Pathways into IC/CS Education and the Workforce». En J. M. Cohoon y W. Aspray (Eds) Women and Information Technology: Research on Under-Representation. Massachussets, MIT Press, 2006, pp 239-278. 
Kennedy, Tracy; Wellman, Barry y Klement, Kristine. «Gendering the digital divide». It and Sociedy. Vol. 1, no 5 (2003), pp. 72-96.

LANDSTROM, Catharina. «Queering feminist technology studies». Feminist Theory vol. 8, no 7 (2007), pp. 7-26.

LA SALLE e ICSA. Estudi DonaTic:Una primera reflexió sobre la dona tecnològica. Barcelona, 2007 <http://www.salle.url.edu/donaTIC/index.html>, consultado el 15-10-2009.

LIFF, Sonia y SHEPHERD, Adrian. «An evolving gender digital divide?». Oxford Internet Institute. Internet Issue Brief. No 2 (2004), pp 1-10.

MERI, Thomas. «Highly qualified workers in science and technology». Statistics in focus, $n^{\circ} 103$ (2007) <http://epp.eurostat.ec.europa.eu/cache/ity_offpub/ ks-sf-07-103/en/ks-sf-07-103-en.pdf>, consultado el 16-10-2009.

Norris, Pippa. Digital Divide. Civic Engagement. Information Poverty, and the Internet Worldwide. Cambridge, Cambridge University Press, 2001.

PÉREZ SAlaZAR, Gabriel. «El estado del arte de la brecha digital», en D. Crovi (coord.) Hacia la sociedad de la información y el conocimiento. México, FCPy UNAM, 2004, pp 137-145.

Plant, Sadie. Zeros + Ones. Digital women + the new technoculture. Nueva York, Doubleday, 1997.

Rasmusen, Bente; HAPNES, Tove. «Excluding Women from Technologies of the future? A case Study of the Culture of Computer Science», en P. Hopkins (ed.) Sex/Machine. Readings in Culture, Gender and Technology. Indiana, Indiana Unversity Press, 1998, pp. 381-394.

SAInZ, Milagros; CASTAÑO, Cecilia y ARTAL, Margarita. Anàlisi del concepte d'alfabetització digital i de les seves implicacions en l'estudi de la divisió digital del gènere. IN3.WP08-001. 2008. <http://www.uoc.edu/in3/dt/cat/sainzcastano-artal.html>, consultado el 15-10-2009.

SCHWARTZ, Ruth «From Virginia Dare to Virginia Slims: Women and Technology in American Life». Technology and Culture 20, 1 (1979), pp. 51-63.

STANLEY, Autumm «Women Hold Up Two-Thirds of the Sky: Notes for a Revised History of Technology». En P. Hopkins (ed.) Sex/Machine. Readings in Culture, Gender and Technology. Indiana, Indiana University Press, 1998, pp. 17-32.

SVENINGSON, Malin; SundEN, Jenny. Cyberfeminism in Northern Lights: Digital Media and Gender in a Nordic Context. Cambridge, Cambridge Scholars Publishing, 2007.

TURKLE, Sherry. «Computational reticence. Why women fear the intimate machine». En P. Hopkins, P (ed.) Sex/Machine. Readings in Culture, Gender and Technology. Indiana, Indiana University Press, 1998, pp. 365-380.

Wajcman, Judy. Feminism confronts technology. Pennsylvania, The Pennsylvania State University Press, 1991. 
- «Reflections on Gender and Technology Studies: In What State is the Art?» Social Studies of Science, Vol. 30, no 3 (2000), pp. 447-464.

- El tecnofeminismo. Madrid, Ediciones Cátedra, 2006.

Wajcman, Judy y Pham LoBB, Le Ahn «The Gender Relations of Software Work in Vietnam». Gender Technology and Development. Vol. 11, no 1 (2007), pp. 1-26. WajCman, Judy. «Feminist Theories of Technology». Cambridge Journal of Economics Advance Access. doi:10.1093/cje/ben057, (2009), pp. 1-10.

WILLEN, Hakan. «Measuring gender differences among Europe's knowledge workers». Statistics in Focus $n^{\circ} 12$ (2006). <http://epp.eurostat.ec.europa.eu/cache/ ity_offpub/ks-ns-06-012/en/ks-ns-06-012-en.pdf>, consultado el 16-10-2009. 